\title{
Lesões orais em idosos que fazem uso de prótese dentária: um protocolo de revisão de
}

\section{escopo}

Oral lesions in elderly denture wearers: a scoping review protocol

Lesiones orales en los ancianos que utilizan prótesis dentales: un protocolo de revisión de alcance

\author{
Júlio César Guimarães Freire \\ ORCID: https://orcid.org/0000-0002-4287-3278 \\ Universidade Federal da Paraíba, Brasil \\ E-mail: julio.guimaraes@academico.ufpb.br \\ Jéssyka Hellem de Melo Pereira \\ ORCID: https://orcid.org/0000-0002-7367-0107 \\ Universidade Federal da Paraíba, Brasil \\ E-mail: jessyka.hellem@academico.ufpb.br \\ Paulo Henrique Lucas do Nascimento \\ ORCID: https://orcid.org/0000-0003-4281-6844 \\ Universidade Federal da Paraíba, Brasil \\ E-mail: paulo.lucas@academico.ufpb.br \\ Pedro Antônio Medeiros da Silva \\ ORCID: https://orcid.org/0000-0002-2637-7231 \\ Universidade Federal da Paraíba, Brasil \\ E-mail: pams2@academico.ufpb.br \\ Aparecida Borges \\ ORCID: https://orcid.org/0000-0003-0753-9423 \\ Universidade Federal da Paraíba, Brasil \\ E-mail: ab@academico.ufpb.br \\ Eduarda Gomes Onofre de Araújo \\ ORCID: https://orcid.org/0000-0001-7107-6107 \\ Universidade Federal da Paraíba, Brasil \\ E-mail: eduarda.onofre@academico.ufpb.br \\ Gabrieli Duarte Farias \\ ORCID: https://orcid.org/0000-0001-6904-2856 \\ Universidade Federal da Paraíba, Brasil \\ E-mail: gabrieli.duarte@academico.ufpb.br \\ Carmem Silvia Laureano Dalle Piagge \\ ORCID: https://orcid.org/0000-0001-7999-2943 \\ Universidade Federal da Paraíba, Brasil \\ E-mail: carmem.piagge@academico.ufpb.br \\ Cláudia Batista Mélo \\ ORCID: https://orcid.org/0000-0001-5300-3510 \\ Universidade Federal da Paraíba, Brasil \\ E-mail: claudia.melo@academico.ufpb.br
}

\begin{abstract}
Resumo
O envelhecimento da população faz surgir a necessidade de cuidados especiais com a saúde. O edentulismo, por exemplo, é uma condição bucal que afeta, principalmente, os idosos, sendo comum o uso de próteses dentárias que muitas vezes geram lesões orais. Este protocolo propõe uma revisão de escopo sobre a prevalência de lesões na mucosa oral em decorrência do uso de prótese dentária na população idosa. Desse modo, o objetivo do referido protocolo é apresentar a metodologia que será adotada, de acordo com as recomendações de estruturação do Joanna Briggs Institute para revisões de escopo, sendo este documento previamente registrado no repositório Open Science Framework. A estratégia de busca será elaborada de modo a identificar as possíveis fontes de evidências publicadas nas seguintes bases de dados: Cochrane, PubMed via MEDLINE, LILACS, Web of Science e Scopus. No que se refere à literatura cinzenta, serão feitas buscas através do Google Scholar. Serão utilizados os seguintes descritores do vocabulário controlado Descritores em Ciências da Saúde/Medical Subject Headings: idoso/aged, prótese dentária/dental prosthesis e manifestações bucais/oral manifestations, seus sinônimos e variações ortográficas. A estratégia PCC (População, Conceito, Contexto) servirá de base para elaboração da pergunta de pesquisa e definição dos critérios de inclusão e exclusão. Os resultados da revisão de escopo proposta deverão mostrar os fatores que influenciam a prevalência de lesões em mucosa oral de pessoas idosas que fazem uso de prótese dentária.
\end{abstract}

Palavras-chave: Protocolos; Revisão; Idoso; Prótese dentária; Manifestações bucais. 


\begin{abstract}
The aging of the population raises the need for special health care. Edentulism, for example, is an oral condition that affects mainly the elderly, and it is common to use dental prostheses that often generate oral lesions. This protocol proposes a scoping review on the prevalence of oral mucosa lesions due to the use of dental prostheses in the elderly population. Thus, the objective of this protocol is to present the methodology that will be adopted, according to the recommendations of the Joanna Briggs Institute for scoping reviews, and this document was previously registered in the Open Science Framework repository. The search strategy will be designed to identify possible sources of evidence published in the following databases: Cochrane, PubMed via MEDLINE, LILACS, Web of Science, and Scopus. With regard to the grey literature, searches will be made via Google Scholar. The following controlled vocabulary descriptors will be used Health Science Descriptors/Medical Subject Headings were used: aged/idoso, dental prosthesis/prótese dentária and oral manifestations/manifestações bucais, their synonyms and spelling variations. The PCC (Population, Concept, Context) strategy will serve as the basis for developing the research question and defining the inclusion and exclusion criteria. The results of the proposed scoping review should show the factors that influence the prevalence of oral mucosal lesions in elderly people who use dental prostheses.
\end{abstract}

Keywords: Protocols; Review; Aged; Dental prosthesis; Oral manifestations.

\title{
Resumen
}

El envejecimiento de la población conlleva la necesidad de una atención sanitaria especial. El edentulismo, por ejemplo, es una condición oral que afecta principalmente a las personas mayores, y es común el uso de prótesis dentales que a menudo generan lesiones orales. Este protocolo propone una revisión de alcance sobre la prevalencia de las lesiones de la mucosa oral debidas al uso de prótesis dentales en la población de edad avanzada. Así, el objetivo de este protocolo es presentar la metodología que se adoptará, según las recomendaciones de estructuración del Instituto Joanna Briggs para las revisiones de alcance, estando este documento previamente registrado en el repositorio Open Science Framework. La estrategia de búsqueda se diseñará para identificar posibles fuentes de evidencia publicadas en las siguientes bases de datos: Cochrane, PubMed vía MEDLINE, LILACS, Web of Science y Scopus. En cuanto a la literatura gris, las búsquedas se harán a través de Google Scholar. Se utilizarán los siguientes descriptores del vocabulario controlado Descriptores en Ciencias de la Salud/Encabezamientos de Materias Médicas: edad/idoso, prótesis dental/prótese dentária y manifestaciones orales/manifestações bucais, sus sinónimos y variaciones ortográficas. La estrategia PCC (Population, Concept, Context) servirá de base para elaborar la pregunta de investigación y definir los criterios de inclusión y exclusión. Los resultados de la revisión de alcance propuesta deben mostrar los factores que influyen en la prevalencia de las lesiones de la mucosa oral en las personas mayores que utilizan prótesis dentales.

Palabras clave: Protocolos; Revisión; Anciano; Prótesis dental; Manifestaciones bucales.

\section{Introdução}

Em face da transição demográfica, há alguns anos, observa-se um crescente aumento da população idosa em decorrência da diminuição na taxa de fecundidade e do aumento na expectativa de vida (Luchesi, 2017; Da Silva Jr. et al., 2021). Para compreender essa nova dinâmica do perfil sociodemográfico mundial, são necessárias mudanças nos sistemas de saúde a fim de assistir de maneira eficaz esses indivíduos.

Nesse contexto, as doenças crônicas que acometem a maior faixa etária da população precisam ser tratadas e acompanhadas, porém, as dificuldades para o agendamento de consultas médicas e odontológicas e as elevadas taxas de ocupação dos serviços de saúde, sobretudo, públicos, vêm prejudicando o acesso integral à saúde dos idosos (Rizzuto et al., 2017; Silva et al., 2018; Araújo \& Riatto, 2020).

Uma das principais condições bucais observadas da pessoa idosa é o edentulismo, isto é, a perda total ou parcial de alguns elementos dentários (Dantas, 2018; Souza et al., 2019). Presente na maioria dos idosos brasileiros, sua ocorrência causa dificuldades na mastigação e, consequentemente, problemas digestivos, além da redução da autoestima, com risco ao desencadeamento de problemas psicológicos e implicações na qualidade de vida do indivíduo (Nascimento et al., 2019; Pena et al., 2019). A fim de recuperar as funções mastigatórias e a autoestima do idoso, é necessário o uso de próteses dentárias, as quais podem ser totais ou parciais, fixas ou removíveis (Azevedo et al., 2017; Cunha et al., 2018).

Devido a múltiplos fatores relacionados, principalmente, à idade, muitos idosos desconhecem os riscos oriundos do uso de prótese dentária. A confecção desse tipo de prótese deve considerar diversos elementos importantes, no que se refere à técnica de acordo com as condições clínicas e radiográficas que, caso seja inadequada, pode levar ao aparecimento de lesões na mucosa 
oral devido à má adaptação da prótese (Trindade et al., 2018; Silva et al., 2019).

Outro fator relevante com relação ao uso das próteses dentárias, é a má higienização da cavidade bucal, que também pode desencadear lesões na mucosa oral. Muitas vezes, a falta de instrução do idoso e/ou do seu cuidador e a orientação inadequada realizada pelo cirurgião-dentista provocam diversas manifestações, tais como: estomatite protética, queilite angular, hiperplasia fibrosa e úlcera traumática (Cunha et al., 2015; Cunha et al., 2016; Silva et al., 2018; Choufani et al., 2020).

Diante do exposto, esse protocolo define os passos que serão seguidos na revisão de escopo, a qual buscará responder o seguinte questionamento: “Qual a prevalência de lesões orais em idosos que fazem uso de prótese dentária?". Portanto, o estudo terá como objetivo principal identificar, por meio de uma revisão de literatura, a prevalência de lesões na mucosa oral de idosos que fazem uso de prótese dentária.

\section{Metodologia e Resultados}

\subsection{Tipo de estudo}

Trata-se de um protocolo para a realização de uma revisão de escopo, que será conduzida de acordo com as recomendações de estruturação do Manual do Instituto Joana Briggs, cujo método visa mapear os principais conceitos, clarificar e identificar lacunas do conhecimento, devendo-se obedecer às seguintes etapas: 1) elaboração da questão de pesquisa e definição dos descritores de busca; 2) pesquisa da literatura em bases de dados internacionais; 3) leitura dos títulos e resumos dos artigos para seleção de acordo com critérios de inclusão e exclusão; 4) leitura na íntegra dos estudos selecionados e mapeamento dos dados; 5) sumarização e análise crítica dos resultados; 6) apresentação dos principais resultados (Peters et al., 2020), sendo o referido protocolo previamente registrado na Open Science Framework com o número de registro DOI 10.17605/OSF.IO/MC8E5 a fim de garantir a transparência da revisão.

\subsection{Pergunta norteadora e Critérios de inclusão}

A pergunta norteadora da pesquisa englobará os elementos do acrônimo PCC (Participantes, Conceito, Contexto), que orientam a definição dos critérios de inclusão para a revisão:

- Qual a prevalência de lesões orais em idosos que fazem uso de prótese dentária?

\subsubsection{Participantes}

Estudos clínicos com acesso livre, conduzidos em pacientes idosos (idade igual ou superior a 60 anos), sem distinção de sexo ou etnia, que fazem uso de prótese dentária (fixa/removível, total/parcial) diagnosticados com alguma lesão oral relacionada ao uso da prótese.

\subsubsection{Conceito}

A existência de evidência científica que associe a ocorrência de lesões orais ao uso de prótese dentária na população idosa. Assim, estudos que analisaram a prevalência das referidas manifestações serão considerados para compor a revisão de escopo.

\subsubsection{Contexto}

O contexto abrangerá todas as fontes de evidência existentes na literatura mundial. Desse modo, nenhum contexto específico será determinado para refinar o escopo da revisão. 


\subsection{Estratégia de busca e Fontes de informação}

A estratégia de busca será elaborada de modo a identificar as possíveis fontes de evidências publicadas nas diversas bases de dados. Para isso, serão elencados os principais assuntos e os termos que os representam. Junto de cada assunto serão selecionados sinônimos, variações ortográficas e formas no singular/plural. Para uma maior abrangência na captura dos estudos, serão consultados os descritores, em português e inglês, e seus sinônimos das Ciências da Saúde pelo DeCS/MeSH (Descritores em Ciências da Saúde/Medical Subject Headings), fazendo-se uso de operadores booleanos para elaboração da estratégia de busca. No Quadro 1, estão divididos os assuntos e seus sinônimos, sendo apresentada a estratégia de busca completa, em português e em inglês, para as respectivas bases de dados.

A partir da estratégia de busca, serão realizadas pesquisas com o objetivo de localizar os estudos publicados sobre a temática, além de selecionar artigos relevantes como base para a revisão. Tais buscas serão realizadas nas seguintes bases de dados: Cochrane, PubMed via MEDLINE, LILACS (Literatura Latino-Americana e do Caribe em Ciências da Saúde), Web of Science e Scopus. No que se refere à literatura cinzenta, serão feitas buscas através do Google Scholar.

Quadro 1. Assuntos e sinônimos utilizados na estruturação da estratégia de busca.

\begin{tabular}{|c|c|c|}
\hline $\begin{array}{l}\text { ASSUNTOS E } \\
\text { ESTRATÉGIA }\end{array}$ & $\begin{array}{l}\text { ASSUNTO E SINÔNIMOS EM } \\
\text { PORTUGUÊS (DeCS) }\end{array}$ & $\begin{array}{c}\text { ASSUNTO E SINÔNIMOS EM INGLÊS } \\
\text { (MeSH) }\end{array}$ \\
\hline ASSUNTO 1 & "idoso" OR “idosos" OR "saúde do idoso" & $\begin{array}{l}\text { "elderly" OR "elderlies" OR "senior" OR } \\
\text { "health of the elderly" OR "aged" }\end{array}$ \\
\hline ASSUNTO 2 & $\begin{array}{l}\text { "prótese dentária" OR "próteses dentárias" } \\
\text { OR "dentadura" OR "dentaduras" OR } \\
\text { "prótese removível” OR "próteses } \\
\text { removíveis" OR "prótese parcial } \\
\text { removível" }\end{array}$ & $\begin{array}{l}\text { "dental prostheses" OR "dental prosthesis" } \\
\text { OR "dental prosthetic" OR "dental } \\
\text { prosthetics" OR "denture" OR "dentures" } \\
\text { OR "denture, partial, removable" }\end{array}$ \\
\hline ASSUNTO 3 & $\begin{array}{l}\text { "lesão" OR "lesões" OR "ferida" OR } \\
\text { "feridas" OR "ferimento" OR "ferimentos" } \\
\text { OR "patologia bucal" }\end{array}$ & $\begin{array}{l}\text { "lesion" OR "lesions" OR "injury" OR } \\
\text { "injuries" OR "wounds and injuries" OR } \\
\text { "pathology, oral" }\end{array}$ \\
\hline $\begin{array}{l}\text { ESTRATÉGIA DE BUSCA } \\
\text { EM INGLÊS - Cochrane, } \\
\text { Pubmed, Web of Science, } \\
\text { Scopus }\end{array}$ & \multicolumn{2}{|c|}{$\begin{array}{l}\text { ("elderly" OR "elderlies" OR "senior" OR "health of the elderly" OR "aged") AND } \\
\text { ("dental prostheses" OR "dental prosthesis" OR "dental prosthetic" OR "dental } \\
\text { prosthetics" OR "denture" OR "dentures" OR "denture, partial, removable") AND } \\
\text { ("lesion" OR "lesions" OR "injury" OR "injuries" OR "wounds and injuries" OR } \\
\text { "pathology, oral") }\end{array}$} \\
\hline $\begin{array}{l}\text { ESTRATÉGIA DE BUSCA } \\
\text { EM PORT/INGLÊS - } \\
\text { LILACS/BVS }\end{array}$ & \multicolumn{2}{|c|}{ 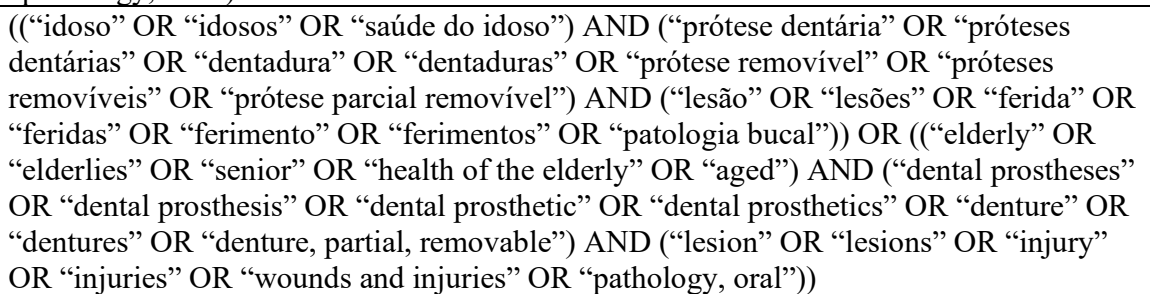 } \\
\hline
\end{tabular}

Fonte: Autores (2021).

\subsection{Seleção de estudos}

Após a execução das buscas nas bases de dados supracitadas, os estudos serão exportados para o software Rayyan (Ouzzani et al., 2016) e, em seguida, para o gerenciador de referências Mendeley (Moraes, 2018). Por meio dessas ferramentas, os artigos serão agrupados para exclusão automática dos arquivos duplicados e, em seguida, serão conduzidas duas fases para seleção dos estudos.

Na primeira fase (triagem), os estudos serão selecionados com base na leitura e análise do título e do resumo; já na segunda fase (seleção propriamente dita), será realizada a leitura completa dos artigos incluídos, considerando os critérios de 
inclusão previamente estabelecidos neste protocolo. Essas etapas serão conduzidas por dois revisores independentes, duplocego, e as divergências existentes serão resolvidas por consenso ou por um terceiro revisor.

Na versão final da revisão de escopo, será apresentado um fluxograma que detalhará os resultados das bases de dados, remoção de duplicatas, triagem e seleção dos estudos, além dos motivos de exclusão. Ademais, o Checklist PRISMA-ScR será utilizado para a elaboração da referida revisão de escopo (Tricco et al., 2018).

\subsection{Extração de dados}

Os dados serão extraídos a partir dos estudos selecionados para compor a revisão de escopo, sendo esta etapa também executada de modo independente por dois revisores, que utilizarão um formulário desenvolvido pelos próprios autores (Quadro 2). Os dados extraídos incluirão informações específicas sobre as fontes de evidência, tais como: autor/ano, local, título, tipo de publicação, objetivo do estudo, método, população e amostra, além dos principais resultados relacionados à pergunta de pesquisa. Qualquer alteração no formulário poderá ser realizada e será descrita na versão final da revisão.

Quadro 2. Formulário de extração de dados.

\begin{tabular}{|c|c|}
\hline INFORMAÇÕES EXTRAÍDAS & DETALHES \\
\hline \multicolumn{2}{|l|}{ Informações bibliográficas } \\
\hline Autores; Ano & Sobrenome dos autores; Ano da publicação \\
\hline Local & País onde o estudo foi realizado \\
\hline Título & Título da publicação (no idioma original) \\
\hline Tipo de publicação & Tese, dissertação ou artigo \\
\hline \multicolumn{2}{|l|}{ Características do estudo } \\
\hline Objetivo & Objetivo principal da pesquisa \\
\hline Método & Método de abordagem quantitativo e/ou qualitativo \\
\hline População e amostra & Descrição da população e da amostra do estudo \\
\hline Principais resultados & $\begin{array}{l}\text { Resultado principal do estudo (tipo de prótese, lesões } \\
\text { associadas e suas características, etc.) }\end{array}$ \\
\hline
\end{tabular}

Fonte: Adaptado de Paiva et al. (2021).

\subsection{Análise e apresentação dos dados}

Os dados obtidos serão apresentados em forma de diagramas, imagens, quadros ou tabelas de modo que estejam alinhados com o objetivo da revisão, sendo acompanhados de uma descrição com o objetivo de facilitar a compreensão do leitor. Os resultados deverão mostrar os fatores que influenciam a prevalência de lesões em mucosa oral de pessoas idosas, colocando em evidência as características da respectiva condição.

\subsection{Aspectos Éticos}

A revisão de escopo proposta utilizará referências bibliográficas disponíveis publicamente e, portanto, não necessitará da aprovação ética prévia de Comitê de Ética em Pesquisa. Além disso, todos os autores envolvidos com o estudo não estarão vinculados a instituições financiadoras, sendo inexistente qualquer conflito de interesse.

\section{Considerações Finais}

Pode-se inferir que o objetivo do atual protocolo de revisão de escopo foi alcançado por meio da sua própria elaboração, que sistematizou os procedimentos necessários para a produção futura de uma revisão de escopo de acordo com o manual do Instituto Joanna Briggs. Todavia, para trabalhos futuros, sugere-se a construção de outros protocolos que subsidiem a execução de revisões de literatura, com metodologias consistentes, sobretudo, em relação à temática manifestação de lesões orais em idosos 
que fazem uso de prótese dentária.

\section{Referências}

Araújo, C. K. C. P., \& Riatto, S. G. (2020). Tratamento odontológico de pacientes geriátricos com doenças sistêmicas. Revista Diálogos em Saúde, 3 (2), 55-71.

Azevedo, J. S., Azevedo, M. S., Oliveira, L. J. C., Correa, M. B., \& Demarco, F. F. (2017). Uso e necessidade de prótese dentária em idosos brasileiros segundo a Pesquisa Nacional de Saúde Bucal (SBBrasil 2010): prevalências e fatores associados. Cadernos de Saúde Pública, 33 (8), e00054016. https://doi.org/10.1590/0102-311X00054016

Choufani, A., Folliguet, M., Chahine, N., Rammal, S., \& Doumit, M. (2020). Prevalence of Oral Mucosal Lesions Among the Institutionalized Elderly Population in Lebanon. Gerontology and Geriatric Medicine. https://doi.org/10.1177/2333721420925189

Cunha, A. S. S., Cyrino, R. F., Dias, M. L., \& Leite, J. J. G. (2015). Biofilmes de Candida spp. em próteses removíveis usadas por pacientes idosos: uma revisão narrativa da literatura. Revista Diálogos Acadêmicos, 4 (2), 109-114. http://revista.fametro.com.br/index.php/RDA/article/view/96

Cunha, M. A. G. M., Matta-Machado, A. T. G., Lucas, S. D., \& Abreu, M. H. N. G. Availability of Dental Prosthesis Procedures in Brazilian Primary Health Care. BioMed Research International, ID 4536707. https://doi.org/10.1155/2018/4536707

Cunha, A. S. S., Cyrino, R. F., Dias, M. L., \& Leite, J. J. G. (2016). Elaboração de uma cartilha educativa para higienização de próteses odontológicas removíveis em idosos. Revista Diálogos Acadêmicos, 5 (2), 107-113. http://revista.fametro.com.br/index.php/RDA/article/view/125

Dantas, L. R. O. (2018). Autopercepção de idosos atendidos na Atenção Básica sobre a relação edentulismo e envelhecimento. Revista Educação em Saúde, 6 (2), 1-7. https://core.ac.uk/download/pdf/234552404.pdf

Da Silva Júnior, J. B., Rowe, J. W., \& Jauregui, J. R. (2021). Healthy aging in the Americas. Revista panamericana de salud publica. Pan American journal of public health, 45, e116. https://doi.org/10.26633/RPSP.2021.116

Luchesi, G. (2017). Envelhecimento populacional: perspectivas para o SUS. In: Pinheiro, A. et al. Brasil 2050: desafios de uma nação que envelhece. Série Estudos Estratégicos, nº 8. Brasília, DF: Edições Câmara, 2017.

Moraes, T. C. C. (2018). Mendeley: manual do usuário. Piracicaba: ESALQ, 2018.

Nascimento, J. E., Magalhães, T. A., Souza, J. G. S., Sales, M. S. M., Nascimento, C. O., Lopes Júnior, C. W. X., Ferreira, E. F., \& Martins, A. M. E. B. L. (2019). Associação entre o uso de prótese dentária total e o tipo de serviço odontológico utilizado entre idosos edêntulos totais. Ciência \& Saúde Coletiva, 24 (9), 3345-3356. https://doi.org/10.1590/1413-81232018249.23002017

Ouzzani, M., Hammady, H., Fedorowicz, Z., \& Elmagarmid, A. (2016). Rayyan: a web and mobile app for systematic reviews. Systematic Reviews, 5 (210), 110. https://doi.org/10.1186/s13643-016-0384-4

Paiva, C. C. N., Santos, D. L., Cotrim Junior, D. F., Cabral, L. M. S., Cabral, S. C. S., \& Esparis, I. M. (2021). O Sistema Único de Saúde e seus desafios na implementação da atenção à saúde sexual e reprodutiva para a população LGBTQIA+ no Brasil: Protocolo de revisão de escopo. Research, Society and Development, 10 (3), 1-10. http://dx.doi.org/10.33448/rsd-v10i3.12966

Pena, A. C. A., Santos, D. N. V., Rocha, G. B., Carvalho, T. J., Cadorin, E. S., \& Bezerra, I. M. P. (2019). Edentulismo Parcial: consequências biopsicossociais em adultos e idosos em Rio Branco-Acre em 2019. DêCiência em Foco, 3 (2), 26-46.

Peters, M. D. J., Godfrey, C., McInerney, P., Baldini Soares, C., Khalil, H., \& Parker, D. (2017). Chapter 11: Scoping Reviews. In: Aromataris, E., \& Munn, Z. (Editors). Joanna Briggs Institute Reviewer's Manual. The Joanna Briggs Institute, 2017. https://reviewersmanual.joannabriggs.org/

Rizzuto, D., Melis, R.J.F., Angleman, S., Qiu, C., \& Marengoni, A. (2017). Effect of Chronic Diseases and Multimorbidity on Survival and Functioning in Elderly Adults. Journal of the American Geriatrics Society, 65, 1056-1060. https://doi.org/10.1111/jgs.14868

Silva, A. E. R., Echeverria, M. S., Custódio, N. B., Cascaes, A. M., Camargo, M. B. J., \& Langlois, C. O. (2018). Uso regular de serviços odontológicos e perda dentária entre idosos. Ciência \& Saúde Coletiva, 23 (12), 4269-4276. https://doi.org/10.1590/1413-812320182312.30562016

Silva, E. A., Brandão, M. C. V., Freitas, N. B. B. S., Costa, M. C. B., Lima, K. G. D., \& Fernandes, D. C. (2018). Principais lesões bucais em idosos que utilizam prótese dentária: revisão integrativa. Caderno de Graduação, UNIT, 4 (3), 23. https://periodicos.set.edu.br/fitsbiosaude/article/view/4916

Silva, J. R. T. C., Andrade Júnior, J. C. B., Silva, P. H., Teixeira, A. C. O. C., \& Borges-Paluch, L. R. (2019). Lesões bucais decorrentes do uso de próteses dentárias removíveis. Revista Baiana de Saúde Pública, 43 (1), 165-179. https://doi.org/10.22278/2318-2660.2019.v43.n1.a3070

Souza, F. E. P., Alves, D. A., Moreira, F. T. L. S., \& Albuquerque, G. A. (2019). Edentulismo e qualidade de vida: percepção de pacientes da terceira idade. Revista e-Ciência, 7 (2), 5-11. https://doi.org/10.19095/rec.v7i2.428

Tricco, A. C., Lillie, E., Zarin, W., O'Brien, K. K., Colquhoun, H., Levac, D., Moher, D., Peters, M., Horsley, T., Weeks, L., Hempel, S., Ak1, E. A., Chang, C., McGowan, J. et al. (2018). PRISMA extension for scoping reviews (PRISMA-ScR): checklist and explanation. Annals of Internal Medicine, 169 (7), 467-473. https://doi.org/10.7326/M18-0850

Trindade, M. G. F., Oliveira, M. C., Prado, J. P., \& Santana, L. L. P. (2018). Lesões Associadas à má Adaptação e má Higienização da Prótese Total. Id on Line Revista Multidisciplinar e de Psicologia, 12 (42), 956-968. https://doi.org/10.14295/idonline.v12i42.1377 